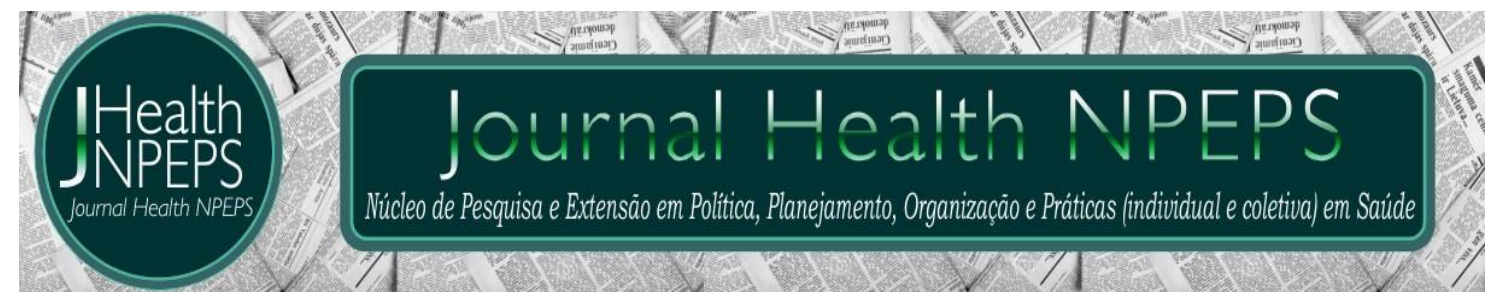

http://dx.doi.org/10.30681/252610105088

ARTIGO ORIGINAL

\title{
Autoavaliação da promoção da autonomia dos idosos: um estudo Delphi
}

\section{Self-assessment of promoting the autonomy of the elderly: a Delphi study}

Autoevaluación de la promoción de la autonomía de las personas mayores: un estudio Delphi

\section{Andreia Maria Novo Lima1, Maria Manuela Ferreira da Silva Martins², Maria Salomé Martins Ferreira ${ }^{3}$, Carla Sílvia Fernandes ${ }^{4}$, Soraia Dornelles Schoeller ${ }^{5}$, Tânia Marisa Pinto Rodrigues ${ }^{6}$, Vítor Sérgio Oliveira Parola ${ }^{7}$}

\section{RESUMO}

Objetivo: validar o conteúdo de um instrumento de autoavaliação da promoção da autonomia dos idosos. Método: estudo qualitativo, com recurso ao método Delphi, com uma amostra de 15 experts, enfermeiros especialistas em enfermagem de reabilitação, enfermagem comunitária, enfermagem de saúde mental e psiquiatria, e enfermagem médico-cirúrgica, com experiência em idosos, experiência clínica e de investigação relevante. Ocorreu no período de junho a setembro de 2020 , com experts da área da saúde do norte de Portugal. Resultados: a análise dos experts confirmou a necessidade de incluir os domínios: fisico, cognitivo, emocional e social, no âmbito das intervenções de enfermagem de avaliação e as intervenções de enfermagem promotoras da autonomia dos idosos. Após a primeira rodada, foram excluídos 34 intervenções, chegando a 68 intervenções de enfermagem para a promoção

\footnotetext{
${ }^{1}$ Enfermeira. Mestre em Enfermagem de Reabilitação. Instituto de Ciências Biomédicas Abel salazar, Escola Superior de Saúde - Fernando Pessoa, CINTESIS. Porto, Portugal. E-mail: alima2358@hotmail.com ORCID ID: https://orcid.org/0000-0001-7535-9040 Autor para correspondência - Endereço: Praça de 9 de Abril 349, 4249-004. Porto, Portugal.

${ }^{2}$ Enfermeira. Doutora em Ciências de Enfermagem. Professora Coordenadora na Escola Superior de Enfermagem do Porto - CINTESIS. Porto, Portugal. E-mail: mmartins@esenf.pt ORCID ID: https://orcid.org/0000-0003-1527-9940

${ }^{3}$ Enfermeira. Doutora em Psicologia da Saúde. Professora no Instituto Politécnico de Viana do Castelo, UICISA. Viana do Castelo, Portugal. E-mail: salomeferreira@ess.ipvc.pt ORCID ID: https://orcid.org/0000-0003-1685-9891

${ }^{4}$ Enfermeira. Doutora em Ciências de Enfermagem. Professora Coordenadora na Escola Superior de Enfermagem do Porto - CINTESIS. Porto, Portugal. E-mail: carlasilviaf@gmail.com ORCID ID: https://orcid.org/0000-0001-7251-5829 ${ }^{5}$ Enfermeira. Doutora em Filosofia da Saúde e Enfermagem. Professora do Programa de Pós-Graduação de Enfermagem da Universidade Federal de Santa Catarina (UFSC). Florianópolis, Santa Catarina, Brasil. E-mail: soraiadornelleschoeller@gmail.com ORCID ID: https://orcid.org/0000-0002-2822-4407

${ }^{6}$ Enfermeira. Enfermeira especialista em Enfermagem de Reabilitação. Centro Hospitalar Universitário São João. Porto, Portugal. E-mail: tmarisaprodrigues@gmail.com ORCID ID: https://orcid.org/0000-0003-4785-3783 ${ }^{7}$ Enfermeiro. Doutor em Ciências de Enfermagem. Professor na Escola Superior de Enfermagem de Coimbra, Unidade de Investigação em Ciências da Saúde: Enfermagem, Centre for Evidence Based Practice: A Joanna Briggs Institute Centre of Excellence. Coimbra, Portugal. E-mail: vitorparola@esenfc.pt ORCID ID: https://orcid.org/0000$\underline{0002-0050-5004}$
}

Este artigo está licenciado sob forma de uma licença Creative Commons Atribuição 4.0 Internacional, que permite uso irrestrito, distribuição e reprodução em qualquer meio, desde que a publicação original seja corretamente citada. 
da autonomia do idoso. Conclusão: este estudo Delphi revelou eficaz na validação do conteúdo desse instrumento, nomeado por Escala de Autoavaliação da Promoção da Autonomia dos Idosos, que será posteriormente testada em uma população maior.

Descritores: Autonomia Pessoal; Cuidados de Enfermagem; Promoção da Saúde; Idoso.

\section{ABSTRACT}

Objective: to validate the content of a self-assessment instrument to promote the autonomy of the elderly. Method: qualitative study, using the Delphi method, with a sample of 15 experts, specialist nurses in rehabilitation nursing, community nursing, mental health nursing and psychiatry, and medical-surgical nursing, with experience in the elderly, clinical experience and relevant research. It took place from June to September 2020, with health experts from the north of Portugal. Results: the experts' analysis confirmed the need to include the domains: physical, cognitive, emotional and social, within the scope of nursing assessment interventions and nursing interventions that promote the autonomy of the elderly. After the first round, 34 interventions were excluded, reaching 68 nursing interventions to promote the autonomy of the elderly. Conclusion: this Delphi study proved to be effective in validating the content of the instrument, called the Self-Assessment Scale for the Promotion of Elderly Autonomy, which will later be tested in an extended population.

Descriptors: Personal Autonomy; Nursing Care; Health Promotion; Aged.

\section{RESUMEN}

Objetivo: validar el contenido de un instrumento de autoevaluación para promover la autonomía de las personas mayores. Método: estudio cualitativo, utilizando el método Delphi y una muestra de 15 experts, enfermeros especialistas en enfermería de rehabilitación, enfermería comunitaria, enfermería en salud mental y psiquiatría y enfermería médicoquirúrgica, con experiencia en ancianos, experiencia clínica investigación e investigación relevante. Tuvo lugar de junio a septiembre de 2020 , con experts en salud del norte de Portugal. Resultados: the experts' analysis confirmed the need to include the domains: physical, cognitive, emotional and social, within the scope of nursing assessment interventions and nursing interventions that promote the autonomy of the elderly. After the first round, 34 interventions were excluded, reaching 68 nursing interventions to promote the autonomy of the elderly. Conclusion: this Delphi study proved to be effective in validating the content of this instrument, named the SelfAssessment Scale for the Promotion of Elderly Autonomy, which will later be tested in a larger population.

Descriptors: Personal Autonomy; Nursing care; Health promotion; Elderly.

INTRODUÇÃO
A autonomia implica o dever de respeitar a capacidade de decisão e a capacidade de 
execução, e envolve aspectos que se completam na íntegra, considerando-se essencial a promoção de condições, pelos profissionais de saúde que promovam o seu exercicio¹. Este conceito compreende várias dimensões, nomeadamente a biológica, a social, a psicológica e a espiritual, sendo fundamental a sua integração, quando se pretende abranger e trabalhar todo o seu âmbito².

No contexto de prestação de cuidados, assiste-se diariamente à aplicação do conceito de autonomia por parte dos enfermeiros e de outros profissionais de saúde se referindo apenas à independência física e funcional da pessoa ${ }^{3,4}$. Simultaneamente nos processos de enfermagem, prescrevem-se intervenções que visam a promoção da autonomia, mas quando analisada a sua aplicabilidade, constata-se que se relacionam à promoção da independência 5 .

A maioria dos profissionais se concentra nos aspectos legais, como a proteção de um direito ou o cumprimento de um dever, do que nos aspectos pessoais e intrínsecos procedentes da capacidade de os pacientes decidirem autonomamente ${ }^{6}$. Esta decisão autonoma é movida pela motivação de dominar o que é seu e o seu destino, ou seja, controlar a própria vida, através da liberdade de escolha e o seu comportamento, pelas sua ações ${ }^{2,7}$.

Para a efetiva concretização da autonomia, tornase necessário o desenvolvimento intelectual, a capacidade de gestão das emoções, a adaptação à condição física e o envolvimento social através do empoderamento ${ }^{8}$. Garantidas estas condições, estará também promovida a autonomia e concomitantemente a qualidade de vida e a dignidade humana 9 .

No idoso, a autonomia assume ainda maior relevância, já que pelos processos decorrentes do envelhecimento, a pessoa idosa é colocada numa situação de vulnerabilidade, não só fisica, mas também intelectual e cognitiva ${ }^{10}$. Tal como, tem-se observado um aumento significativo da população idosa em Portugal e no mundo, o que tem provocado e continua a realçar as preocupações no que concerne às condições de vida e os 
processos de saúde-doença dessas pessoas $^{11}$. Assim, face ao risco de vulnerabilidade e fragilidade associados ao processo de envelhecimento, a autonomia do idoso é um conceito largamente utilizado na disciplina de enfermagem, porém, esta não possui nenhum instrumento que seja capaz de permitir aos enfermeiros

percecionarem/analisarem a forma como trabalham a autonomia dos idosos, na sua prática clínica, efetivando-se através da autoavaliação ${ }^{12}$.

São

necessários

instrumentos de avaliação que permitam aos enfermeiros percecionarem/analisarem os cuidados que prestam, objetivando a melhoria dos cuidados prestados, permitindo uma eficaz avaliação, controle e monitorização, desses mesmos cuidados. A pesquisa efetuada foi realizada tendo por base a questão de investigação: Quais são as intervenções de enfermagem promotoras da autonomia do idoso?. Cientes desta necessidade, o presente estudo tem como objetivo de validar o conteúdo de um instrumento de autoavaliação da autonomia dos idosos.

\section{MÉTODO}

Para identificar os itens da escala, ou seja as intervenções de enfermagem que promovem a autonomia do idoso recorreu-se à metodologia qualitativa, com recurso ao método de Delphi, a qual envolve a seleção de um grupo de especialistas que são consultados através da utilização de um questionário, num processo interativo até chegar a um consenso sobre as respostas ${ }^{13,14}$. A mesma decorreu no período de junho a setembro de 2020, com experts da área da saúde, do norte de Portugal. As diferentes etapas do procedimento são apresentadas na Figura 1.

0 painel de experts que participaram neste estudo foi constítuido por enfermeiros com experiência profissional (formação e experiência clínica) com idosos. Para serem incluídos neste estudo os experts tiveram que atender aos seguintes critérios de inclusão: 1) ter no minimo 2 anos de experiência profissional com idosos; 
e 2) ter atividade clínica e de investigação ativa; e 3) ser detentor do grau de especialista em enfermagem de reabilitação, ou enfermagem comunitária, ou enfermagem de saúde mental e psiquiatria e ou enfermagem médico-cirúrgica.

Os primeiros itens foram criados, através de várias fontes de informação ${ }^{12,15,16}$.

Inicialmente, foi efetuada uma revisão da literatura, recorrendo às bases de dados: Scopus, CINAHL (via EBSCO) e MEDLINE (via PubMed). Como estratégia de pesquisa, foi efetuada uma combinação das seguintes palavras-chave e termos de índice identificados: Patients, Independence, Autonomy, Theory, Concept, Patient Autonomy, Independent Living, utilizando os operadores boleanos NOT, AND e OR, para filtrar ou expandir os artigos com base nos resultados. A pesquisa com recurso às referidas palavras-chave e termos de índice identificados foi adaptada de acordo com as especificidades de cada base de dados. Considerou-se para a inclusão nesta revisão estudos escritos em inglês, espanhol e português, publicados entre 2010 e 2020. Através a pesquisa foram identificados 1519 artigos.

Posteriormente, recorreuse também à análise de conceito efetuada pelos autores ${ }^{15}$, para compreender o âmbito do conceito de autonomia, e à scoping review realizada para analisar os instrumentos utilizados para avaliar a autonomia da pessoa ${ }^{12}$.

Os autores levaram também, a cabo um estudo qualitativo $^{16}$ com 0 objetivo de analisar as práticas dos enfermeiros na promoção da autonomia dos idosos, sendo este também uma das fontes de informação para a realização do presente estudo.

Da análise de todas estas fontes de informação foram identificadas 102 itens ou possíveis intervenções de enfermagem promotoras da autonomia do idoso. Depois destes procedimentos, tornou-se necessário a realização do presente estudo, para obter o consenso por parte dos peritos sobre os itens relevantes a incluir no instrumento.

Num estudo Delphi, o procedimento requer tantas 
Autoavaliação da promoção da autonomia...

rodadas quantas as necessárias para alcançar um consenso entre os especialistas $^{13,14}$. Neste estudo, tal como efetuado em estudos anteriores com objetivos semelhantes ${ }^{17}$, os itens da escala, foram identificados, quando os mesmos obtiveram $75 \%{ }^{18}$ ou mais, do consenso entre os participantes. Foram suficientes duas rodadas, 0 que geralmente é frequente neste tipo de metodologia ${ }^{13}$.

$\mathrm{Na}$ primeira rodada, para além de ser novamente explicado o estudo e os objetivos do mesmo, foram solicitados os dados sociodemográficos, tempo de experiência profissional, tempo de experiência profissional com idosos, idade, sexo, especialização na área de enfermagem e tipo de atividade desempenhada. Foi também enviada a lista de itens ou potenciais intervenções de enfermagem promotoras da autonomia do idoso, na qual os experts selecionavam os itens "concordo" ou "discordo", com possibilidade de inserção de comentários.

Os profissionais foram selecionados com base na sua área de intervenção e/ou investigação, assim como, por serem considerados pessoas de referência nas suas instituições. O contato foi realizado pela equipa de investigação, com cada um destes elementos. De acordo com a literatura ${ }^{18}$, neste tipo de estudos, o grupo de experts, deve ser superior a 10, sendo igualmente, recomendado não exceder os 30 participantes. Assim, foram convidados 23 experts, mas, apenas 15 aceitaram participar. Aqueles que aceitaram participar foram convidados a responder por e-mail no prazo máximo de três semanas. Nesse período foram enviados dois lembretes. A análise, pelos participantes, exigia cerca de 30 minutos para ser realizada.

Os resultados desta rodada foram analisados para verificar a concordância na resposta dos participantes e os respetivos comentários. Assim, efetuou-se uma análise descritiva, com cálculo de frequências e percentagens para as diferentes variáveis do estudo.

Posteriormente, OS participantes foram informados dos resultados da primeira rodada, sendo reenviada a lista retificada dos itens, selecionados e 
Autoavaliação da promoção da autonomia...

identificados. Na segunda rodada foi solicitado o mesmo procedimento da primeira rodada. Mais uma vez, para incentivar a participação, foram enviados dois lembretes durante as três semanas, incentivando o envio da resposta.

Para a análise dos dados, recorreu-se à estatistica descritiva, através do cálculo das frequências e percentagens, bem como a análise de concordância dos itens por rodada, utilizando o programa estatistico SPSS 26.0. Considerou-se como uma taxa de aceitável de concordância, para cada item de $75 \%$, entre os peritos, em todas as rodadas $^{18}$.

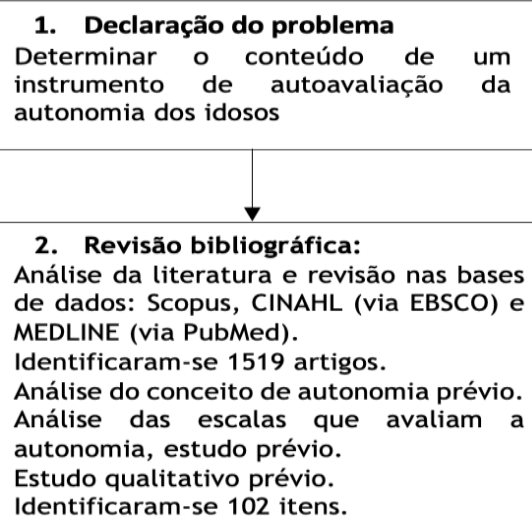

3. Seleção do painel de experts/Critérios de inclusão: No mínimo ter experiência clinica de 2 anos com idosos.

Atividades clínica e de investigação ativa.

Ser enfermeiros especialistas em:

- Enfermagem de reabilitação

- Enfermagem Comunitária

- Enfermagem de saúde mental e psiquiatria

- Enfermagem médico-cirúrgica

4. Rodada 1 do estudo Delphi Elaboração e envio através do e-mail do 1. ${ }^{\circ}$ questionário com a carta de apresentacão do estudo.

apresentacão do estudo.
5. Análise das respostas e
tratamento dos primeiros resultados.
Comunicar os principais resultados aos
participantes.
participantes.

6. Rodada 2 do estudo Delphi Carta de apresentação/lembrete

Elaboração e envio através de e-mail do $2 .^{\circ}$ questionário.

7. Preparação do relatório final com as conclusões do estudo

Figura 1 - Passos do estudo Delphi. Porto, Portugal.

\section{RESULTADOS E DISCUSSÃO}

$\mathrm{Na}$ primeira rodada foram convidados de 23 experts a participar, no entanto, apenas 15 aceitaram participar no estudo. A maioria dos experts participantes são do sexo feminino $(66,7 \%)$; especialistas $(60,0 \%)$, sendo a maioria especialistas em enfermagem de reabilitação $(46,7 \%)$; como uma média de 39,4 
anos de idade, 17,6 anos de

dessa experiência com idosos

experiência profissional e 14,5 anos

(Tabela 1).

Tabela 1 - Caraterísticas sociodemográficas dos experts em cada rodada. Porto, Portugal. 2020. $(n=15)$

N. ${ }^{\circ}$ de participantes convidados: $\mathrm{n}=23$ $1^{\mathrm{a}}$ rodada e $2^{\mathrm{a}}$ rodada Aceitaram participar

Sexo:

Feminino

Masculino

Experiência profissional

\begin{tabular}{cc}
\multicolumn{2}{c}{$1^{\text {a }}$ rodada e $2^{\text {a }}$ rodada } \\
Aceitaram participar \\
$N$ & $\%$ \\
10 & 66,7 \\
5 & 33,3 \\
8-38 $(X=17,6$ anos, & \\
SD $=8,14)$ & \\
& \\
1 & 6,7 \\
5 & 33,3 \\
9 & 60,0 \\
$4-33(X=14,5$ anos, & \\
SD $=6,65)$ & \\
$31-59(X=39,4$ & \\
anos, SD $=7,75)$ & \\
7 & \\
2 & 46,7 \\
2 & 13,3 \\
3 & 13,3 \\
&
\end{tabular}

Tipo de atividade desempenhada:

Professor auxiliar (enfermagem)

Professor adjunto (enfermagem)

Enfermeira especialista

Experiência profisional com idosos

Idade

Especialidade:

Enfermagem de reabilitação

Enfermagem comunitária

Enfermagem de saúde mental e psiquiatria

Enfermagem médico-cirúrgica

0 grupo de experts apresenta uma vasta experiência clínica e/ou de investigação relevante. Foram submetidas a avaliação por parte dos experts, através do questionário, os 102 itens ou possíveis intervenções de enfermagem promotoras da autonomia do idoso, resultantes da pesquisa e dos estudos realizados previamente.

Relativamente, ao objetivo principal deste estudo, o painel de experts concordou que as intervenções de enfermagem podem se dividir em intervenções que permitem a avaliação da autonomia e em intervenções promotoras de autonomia, num total de 68 itens.

Deste modo, dos 102 itens iniciais obteve-se uma concordância superior a 75\% em 68 dos itens.

De acordo, com a opinião dos avaliadores e depois da segunda rodada, as intervenções que permitem a avaliação da autonomia dos idosos efetivam-se no domínio físico, cognitivo, social e emocional (Quadro 1). 
Quadro 1 - Intervenções de enfermagem, no âmbito da avaliação dos domínios: físico, cognitivo, social e emocional. Porto, Portugal. 2020.

\begin{tabular}{|c|c|}
\hline Domínio físico & Domínio cognitivo \\
\hline - Capacidade para ir às compras & $\begin{array}{l}\text { - Capacidade de decisão sobre acontecimentos } \\
\text { concretos (ex: escolher a roupa que quer vestir, } \\
\text { escolher o lugar onde permanecer...) }\end{array}$ \\
\hline - Capacidade para gerir o dinheiro & $\begin{array}{l}\text { - Capacidade de entender as questões que lhe são } \\
\text { colocadas }\end{array}$ \\
\hline - Capacidade para utilizar o telefone & $\begin{array}{l}\text { - Capacidade para responder de acordo com as } \\
\text { questões que lhe são colocadas }\end{array}$ \\
\hline - Capacidade para cozinhar & $\begin{array}{l}\text { - Capacidade para reter as informações } \\
\text { fornecidas }\end{array}$ \\
\hline - Capacidade para utilizar os transportes & $\begin{array}{l}\text { - Conhecimentos sobre as intervenções que } \\
\text { promovem a autonomia }\end{array}$ \\
\hline - Capacidade para as atividades de lazer & Domínio Social \\
\hline - Capacidade para se alimentar & $\begin{array}{l}\text { Capacidade para estabelecer relações } \\
\text { interpessoais }\end{array}$ \\
\hline $\begin{array}{l}\text { - Capacidade para cuidar da higiene } \\
\text { pessoal }\end{array}$ & - Volição para a interação com outros \\
\hline - Capacidade para usar o sanitário & $\begin{array}{l}\text { - Perceção de que os outros respeitam as suas } \\
\text { decisões }\end{array}$ \\
\hline - Capacidade para se erguer & Domínio Emocional \\
\hline - Capacidade para se transferir & $\begin{array}{l}\text { - Capacidade de manifestar as suas emoções } \\
\text { (reação verbal e não verbal, alegria, choro, raiva, } \\
\text { tristeza) }\end{array}$ \\
\hline - Capacidade para se virar & $\begin{array}{l}\text { - Capacidade de responder com emoções } \\
\text { adequadas às situações }\end{array}$ \\
\hline - Capacidade para andar & $\begin{array}{l}\text { - Perceção de compreensão das suas emoções } \\
\text { pelos outros } \\
\text { - Capacidade para compreender as emoções dos } \\
\text { outros }\end{array}$ \\
\hline
\end{tabular}

$\mathrm{Na}$ opinião dos experts e

promoção da autonomia dos idosos depois da segunda rodada, as (Quadro 2). intervenções que permitem a

Quadro 2 - Intervenções de enfermagem promotoras da autonomia do idoso. Porto, Portugal. 2020.

\begin{tabular}{|c|c|}
\hline & \\
\hline $\begin{array}{l}\text { - Estabeleço uma relação empática com o } \\
\text { idoso } \\
\text { - Respeito a privacidade do idoso } \\
\text { - Respeito as crenças e rituais religiosos do } \\
\text { idoso } \\
\text { - Respeito a vontade e as escolhas do idoso } \\
\text { - Explico os procedimentos ao idoso } \\
\text {-Dou tempo ao idoso para ele realizar as } \\
\text { atividades } \\
\text { - Incentivo a independência do idoso }\end{array}$ & $\begin{array}{l}\text { - Capacito o idoso tendo em conta a cognição } \\
\text { - Capacito o idoso para socializar } \\
\text { - Capacito o idoso para a expressão de } \\
\text { emoções } \\
\text { - Promovo a autoestima do idoso } \\
\text { - Executo mobilizações (ativas, ativas- } \\
\text { assistidas, passivas) ao idoso } \\
\text { - Executo exercícios de treino de equilíbrio } \\
\text { ao idoso } \\
\text { - Executo exercícios de treino cognitivo }\end{array}$ \\
\hline
\end{tabular}


- Capacito o idoso para ir às compras

- Capacito o idoso para gerir o dinheiro

- Capacito o idoso para utilizar o telefone

- Capacito o idoso para limpar a casa

- Capacito o idoso para cozinhar

- Capacito o idoso para utilizar os transportes

- Capacito o idoso para atividades de lazer

- Capacito o idoso para o autocuidado: alimentar-se

- Capacito o idoso para o autocuidado: cuidar da higiene pessoal

- Capacito o idoso para o autocuidado: usar o sanitário

- Capacito o idoso para o autocuidado: elevar-se

- Capacito o idoso para o autocuidado: transferir-se

- Capacito o idoso para o autocuidado: virarse

- Capacito o idoso para andar

- Capacito o idoso para o processo de tomada de decisão (jogos de memória) ao idoso

- Ensino/instruo o idoso sobre exercícios de mobilização (ativa, ativa-assistida)

- Treino exercícios de mobilização (ativa, ativa-assistida) com o idoso

- Ensino/instruo o idoso sobre exercícios de treino de equilíbrio

- Treino o idoso em exercícios de treino de equilíbrio

- Ensino/instruo o idoso sobre exercícios de treino cognitivo

- Treino o idoso em exercícios de treino cognitivo

- Ensino cuidador sobre: autocuidados do idoso

- Ensino o cuidador sobre: atividades instrumentais de vida diárias do idoso

- Ensino o cuidador sobre: promoção de estilos de vida saudáveis do idoso

- Ensino o cuidador sobre: medidas preventivas do idoso

- Ensino o cuidador sobre: gestão da terapêutica do idoso

- Ensino o cuidador sobre: gestão da atividade física do idoso

- Ensino o cuidador sobre: promoção da autonomia do idoso

- Ensino o cuidador sobre promoção da independência do idoso
As intervenções de enfermagem que permitem avaliar a autonomia do idoso, orientam os enfermeiros para as necessidades da pessoa e os potenciais problemas, para os quais devem orientar a sua atenção. Para essa avaliação é frequente, estes profissionais recorrerem a instrumentos, como completemento à avaliação clinica $^{19}$. Assim, a implementação destas intervenções permitem ao enfermeiro elaborar os respectivos diagnósticos de enfermagem, e consequentemente

implementação de intervenções promotoras da autonomia. Os itens incluidos, após consenso entre peritos, estão de acordo com outros estudos realizados anteriormente, em que salientam que a autonomia compreende várias dimensões como a biológica, a psicológica e a social ${ }^{8,20,21}$.

No domínio fisico, através das intervenções identificadas é possível avaliar a capacidade da pessoa idosa para a realização das atividades básicas de vida diárias 
Autoavaliação da promoção da autonomia...

ou também definidas como, autocuidado, e as atividades instrumentais de vida diárias ${ }^{5}$. São consideradas intervenções deste domínio, todas aquelas que permitem avaliar a capacidade da pessoa para se: vestir e despir, arranjar, virar, mover, alimentar, usar o sanitário, transferir e para cuidar da higiene pessoal, ou seja, satisfazer as atividades básicas de vida diárias, para além daquelas intervenções que permitem identificar problemas, ou potenciais problemas nas atividades instrumentais de vida diárias, como: cozinhar, ir às compras, telefonar, utilizar os transportes públicos, cuidar da casa e para a realização de atividades de lazer $^{22}$. O processo de envelhecimento, mesmo que normal, produz mudanças biológicas, fisiológicas e anatómicas, que se traduzem em limitações no desempenho das funções corporais na pessoa idosa, pelo que o enfermeiro deve identificá-las, visando ações de prevenção de dependência e incapacidade e da promoção da saúde ${ }^{23}$.

No domínio cognitivo, as intervenções identificadas permitem dar a conhecer ao enfermeiro, a capacidade inteletual, os conhecimentos, a sua consciencialização e os significados da pessoa idosa 24 . Tal como salientam alguns autores ${ }^{2,25}$, a pessoa só poderá tomar decisões e agir conscientemente, expressando capacidade para controlar as próprias vidas, se for detentora de capacidades no domínio cognitivo. Pese embora, o instrumento para o qual se pretende validar 0 conteúdo, faça a subdivisão de cada um dos dominios (físico, cognitivos, social e emocional), todas as necessidades decorrentes destes domínios são interdependentes e estão interrelacionadas, pelo que no seu todo elas dão resposta ao conceito de autonomia $^{15}$, na sua abordagem integral. Interessa portanto, ao enfermeiro perceber a capacidade da pessoa idosa para reter novos conhecimentos e os conhecimentos que a mesmo já detém sobre as temáticas abordadas.

O envelhecimento, acarreta igualmente consequências no domínio cognitivo, pelo que avaliar, diagnosticar e intervir atempadamente, poderá garantir a 
qualidade de vida da pessoa, a sua dignidade

$$
\text { e }
$$

manutenção/promoção

da

autonomia, adequando programa de estimulação cognitiva ${ }^{26}$. Garantida a capacidade cognitiva, o empoderamento da pessoa idosa, com recurso à literacia é uma ferramenta essencial para a satisfação da sua autonomia ${ }^{27}$, considerando-se essencial a memorização a curto, médio e longo prazo. A literacia em saúde, visa a promoção da saúde, sendo largamente utilizada no âmbito dos cuidados de saúde, na medida em que estimula a pessoa a incutir mudanças e práticas saudáveis, reduzindo assim, vulnerabilidades e fragilidades, a que anteriormente estavam sujeitas ${ }^{23}$, mas para que isto seja possível a pessoa deverá deter tais capacidades, quando se procede à respectiva avaliação.

Sendo a autonomia influenciada pela contexto, onde a pessoa se insere, cabe ao enfermeiro, conhecer todos os aspectos da cultura da pessoa, pois estes podem influenciar a sua saúde?

No domínio social, através das intervenções identificadas é possível determinar a integração social, avaliar a vontade da pessoa idosa para o fazer e a percepção que a mesma tem sobre a forma como os outros respeitam as suas opiniões. A autonomia depende também da capacidade que a pessoa tem de viver numa sociedade, através das relações sociais, familiares, estruturais e situacionais $^{25}$. 0 estabelecimento de relações sociais saudáveis, são fontes intrínsecas de motivação para dominar o próprio destino, controlar a sua vida e o seu comportamento, numa dinâmica entre a classe social, a história de vida, a etnia, o gênero, a economia, a política e a cultura, onde na maioria das situações existem relações de poder ${ }^{28,29}$. Estas relações podem colocar em causa a autonomia da pessoa, quando, a família/prestador de cuidados, substituí o idoso, na tomada de decisão e na realização das atividades de vida diárias.

Em várias sociedades, está instituído o hábito de substituir o idoso, inicialmente nas atividades instrumentais de vida diárias e mais tarde nas atividades básicas de vida diárias, por parte dos familiares 
como forma de carinho. Este acontecimento acaba por ser um desafio para o enfermeiro, na medida em que este profissional, reconhece 0 risco desta substituição, estando alerta para uma intervenção precoce, através dos ensinos aos prestadores de cuidados. No meio social, a pessoa necessita se sentir volitivo e senhor das suas próprias ações ${ }^{30}$.

Devido ao excesso de trabalho, entre outros fatores perturbadores da qualidade dos cuidados prestados, também os profissionais de saúde, caem na tentação de substituir o doente, mesmo reconhecendo os malefícios de tal substituição, especialmente quando este está institucionalizados ${ }^{31}$. A substituição das pessoas idosas de acordo com a opinião dos enfermeiros especialistas, acontece, porque a mesma, pelos processos decorrentes do envelhecimento demoram mais tempo na realização da tarefa, tempo esse, que referem não ter, devido ao excesso de atividades e intervenções de enfermagem a realizar durante $o$ turno de trabalho aos doentes atribuídos no plano de trabalho ${ }^{32}$.
No domínio emocional, recorrendo às intervenções de enfermagem identificadas, será possível determinar a forma como a pessoa idosa gere as suas emoções, na medida em que é importante a perceção que a pessoa tem de si mesma, as suas emoções, apelando a uma boa inteligência emocional ${ }^{21}$. Tal como salientam outros autores ${ }^{33}$, os idosos consideram ser importante ter expectitivas face à vida, as quais são concretizadas na satisfação com a mesma, bem como, com a capacidade de se manterem ativos, através da implementação de programas formativos, que visem cuidar da mente, do corpo e das relações sociais.

Depois da implementação das supracitadas intervenções avaliativas, são efetuados os respectivos diagnósticos de enfermagem, que podem ser problemas reais ou potenciais, sendo posteriormente implementadas as intervenções que se denominam como intervenções promotoras da autonomia da pessoa idosa, no presente estudo.

As intervenções promotoras da autonomia da pessoa idosa visam 
manter/promover a capacidade executiva e funcional da pessoas nas atividades básicas de vida diária e nas atividades instrumentais de vida diária, promover essa capacidade através da realização de exercícios musculo-esqueléticos, promover a capacidade de decisão, a capacidade informativa e a narrativa, a cognição, a gestão de emoções e a sociabilização ${ }^{32}$.

$$
\text { Em saúde, }
$$

empoderamento tanto das pessoas cuidadas, como dos seus prestadores de cuidados informais é uma ferramenta essencial, para a promoção da autonomia da pessoa idosa, na medida em que promove a competência para lidar com as questões de saúde e com a vida em geral $^{31}$. No que concerne à autonomia, esta só poderá ser garantida através do desenvolvimento da capacidade inteletual, do envolvimento $\mathrm{e}$ integração social, na gestão das emoções e adaptação à nova condição física, sendo para isso necessário investir na literacia em saúde e no aumento dos conhecimentos sobre estilos de vida saudáveis, que premeiem o envelhecimento ativo e saudável da populaçãão 35 -37.

Relativamente às intervenções de enfermagem promotoras da autonomia, no que concerne ao referido empoderamento, estas são todas aquelas que capacitam, ensinam, instruem e treinam, tanto a pessoa idosa como o cuidador informal. Em suma, quando promovida a autonomia da pessoa idosa, está também garantida a sua qualidade de vida e a sua dignidade ${ }^{38}$.

0 presente estudo possui algumas limitações a serem consideradas para permitir uma ponderação justa dos resultados. Uma das limitações pode ser a representatividade dos participantes, uma vez que se desconhece, se os resultados seriam os mesmos, se a amostra fosse maior ou se os participantes fossem outros. No entanto, o grupo foi constituído por profissionais com uma vasta experiência clínica e/ou de investigação na área do idoso, de modo a minimizar esta limitação.

Apesar das limitações, os resultados fornecem informações sobre as intervenções de 
enfermagem para a promoção da autonomia dos idosos, umas na vertente da avaliação e outras promotoras da autonomia, na sua efetividade. Estes achados, permitem garantir que todos os itens do instrumento, são intervenções que os enfermeiros devem implementar na promoção da autonomia dos idosos, e integrando para cada uma das intervenções uma escala do tipo Likert, de cinco níveis de resposta, podem os enfermeiros efetuar uma autoavaliação da promoção da autonomia dos idosos, através da escala (EAPAI). Nesta escala, o profissional assinala a frequência com que implementa as referidas intervenções.

\section{CONCLUSÃO}

Através deste estudo, inicialmente com 102 itens, o instrumento foi submetido à avaliação dos experts, que em consenso consideram oportuno a inclusão de 68 dos itens, os quais dão resposta aos aspectos que 0 conceito de autonomia incluí como: a capacidade física, a capacidade cognitiva, a inteligência emocional e a integração social. Os itens incluem intervenções de enfermagem, no âmbito da avaliação dos domínios: físico, cognitivo, social e emocional e intervenções de enfermagem promotoras da autonomia do idoso.

Dada

multidimensionalidade do conceito de autonomia, muitos aspectos seriam necessários para serem incorporados neste novo instrumento. A sua elaboração seria inatingível, sem o recurso a uma análise qualitativa prévia. 0 estudo Delphi, revelou-se muito útil na pesquisa avaliativa do instrumento, realçando a importância de considerar a opinião de diferentes experts, resultando numa experiência enriquecedora que dá segurança para continuar a investigação. Este estudo contribui assim, para identificar as principais intervenções de enfermagem que podem contribuir para a promoção da autonomia da pessoa idosa.

Estes achados permitaram aos enfermeiros autoavaliar a forma como promovem a autonomia dos idosos, na sua prática clínica, servindo esta ferramenta para a melhoria continua da qualidade de 
cuidados prestados ao idoso, evidenciando a importância da implementação das respectivas intervenções de enfermagem.

Depois da validação do conteúdo do instrumento, pretende-se agora levar a cabo um estudo que valide $\mathrm{o}$ instrumento intitulado Escala de Autoavaliação da Promoção da Autonomia dos Idosos, na população de enfermeiros de Portugal, para garantir a sua fidelidade $e$ consistência interna.

\section{REFERÊNCIAS}

1. Dzeng E. Habermasian communication pathologies in do-not-resuscitate discussions at the end of life: manipulation as an unintended consequence of an ideology of patient autonomy. Sociol Health Illn. 2019; 41(2):325-42.

2. Bouvet R. The primacy of the patient's wishes in the medical decision-making procedure established by French law. Eur J Health Law. 2018; 25(4):42640.

3. Oberstadt MCF, Esser P, Classen $\mathrm{J}$, Mehnert A. Alleviation of psychological distress and the improvement of quality of life in patients with amyotrophic lateral sclerosis: Adaptation of a short-term psychotherapeutic intervention. Front Neurol. 2018; 9(APR).

4. Lima AMN, Martins MMPS, Ferreira MSM, Fernandes CS, Schoeller S, Coelho ARN, Parola VSO. Concept of Elderly Autonomy: Phenomenological study of the opinion of specialist nurses (in press). Porto Biomed J. 2021.

5. Jacobs G. Patient autonomy in home care: Nurses' relational practices of responsibility. Nurs Ethics. 2019; 26(6):1638-53.

6. Bertilsson A-S, von Koch L, Tham K, Johansson U. Clientcentred $A D L$ intervention after stroke: Significant others' experiences. Scand Occup Ther. 2015; 22(5):377-86.

7. Eassey D, Smith L, Reddel HK, Ryan K. The impact of severe asthma on patients' autonomy: A qualitative study. Health Expect. 2019; 22(3):528-36.

8. Brown SL, Salmon P. Reconciling the theory and reality of shared 
decision-making: A "matching" approach to practitioner leadership. Health Expect. 2019; 22(3):275-83.

9. Henry LM, Rushton C, Beach MC, Faden R. Respect and dignity: a conceptual model for patients in the intensive care unit. Narrat Inq Bioeth. 2015; $5(1): 5-14$.

10. Lima AMN, Ferreira MSM, Martins MMPS, Fernandes CS, Moreira MTF, Rodrigues TMP. Independência funcional e o estado confusional de pessoas sujeitas a programa de reabilitação. J Health NPEPS. 2020; 5(2):145-60.

11. Passos J, Sequeira C, Fernandes L. Focos de enfermagem em pessoas mais velhas com problemas de saúde mental. Referência. 2014; Série $\operatorname{IV}(2): 81-91$.

12. Lima $A M N$, Martins MMPS, Ferreira MSM, Fernandes CS, Schoeller SD, Rodrigues TMP, et al. From the challenge of assessing autonomy to the instruments used in practice: A Scoping Review. Porto Biomed J. No prelo, 2021.
13. Almenara JC, Moro Al. Empleo del método Delphi y su empleo en la insvestigación en cominucation y educación. EDUTEC. 2014; 48(jun):1-16.

14. Godet M. Manuel de prospective stratégique -Tome 2-3ème édition-L'Art et la méthode: Dunod; 2007.

15. Lima AMN, Martins MMPS, Ferreira MSM, Schoeller SD, Parola VSO. 0 conceito multidimensional de autonomia: uma análise conceptual recorrendo a uma scoping review (in press). Referência; 2021.

16. Lima $\mathrm{AMN}$, Martins MMPS, Ferreira MSM, Coelho ARN, Schoeller SD, Parola VSO. Focos e intervenções de enfermagem promotoras da autonomia dos idosos. Rev Gaúch Enferm. No prelo, 2021.

17. Expósito-Vizcaíno S, BurjalésMartí D, Miró J. Factores psicosociales en el dolor oncológico: un estudio Delphi. Rev Soc Esp Dolor. 2019; 26(1):21-30.

18. Marques JBV, Freitas DD. Método Delphi: caracterização e potencialidades na pesquisa 
em Educação. Pro-Posições. 2018; 29:389-415.

19. Gardona RGB, Barbosa DA. Importância da prática clínica sustentada por instrumentos de avaliação em saúde. Rev Bras Enferm. 2018; 71(4):1921-2.

20. Trotter G. Autonomy as selfsovereignty. HEC Forum. 2014; 26(3):237-55.

21. Calheiros MM, Patrício JN, Graça J. Staff and youth views on autonomy and emancipation from residential care: a participatory research study. Eval Program Plann. 2013; 39:57-66.

22. Guo HJ, Sapra A. Instrumental Activity of Daily Living. Treasure Island (FL): StatPearls Publishing; 2021.

23. Clares JWB, Nóbrega MMLD, Guedes MVC, Silva, LDFD, Freitas MCD. ICNP ${ }$ nursing diagnoses, outmomes and interventions for community elderly. Rev Bras Enferm. 2019; 72:191-8.

24. Antoniou T, Ala-Leppilampi K, Shearer D, Parsons JA, Tadrous $M$, Gomes T. Like being put on an ice floe and shoved away: A qualitative study of the impacts of opioid-related policy changes on people who take opioids. Int J Drug Policy. 2019; 66:15-22.

25. Bell JAH, Balneaves LG, Kelly MT, Richardson H. Report on a Delphi process and workshop to improve accrual to cancer clinical trials. Current Oncology. 2016; 23(2):125-30.

26. Santos MT, Flores-Mendoza C. Treino Cognitivp para Idosos: Uma Revisão Sistemática dos Estudos Nacionais. Psico-USF. 2017; 22:337-49.

27. Bennett L, Bergin M, Wells JSG. The social space of empowerment within epilepsy services: The map is not the terrain. Epilepsy Behav. 2016; 56:139-48.

28. Bhola P, Chaturvedi SK. Through a glass, darkly: ethics of mental health practitionerpatient relationships in traditional societies. Int J Cult Mental Health. 2017; 10(3):28597.

29. Krishna LK, Watkinson DS, Beng NL. Limits to relational autonomy--the Singaporean experience. Nurs Ethics. 2015; 22(3):331-40. 
Autoavaliação da promoção da autonomia...

30. Benson JJ, Parker Oliver D, Demiris G, Washington K. Accounts of Family Conflict in Home Hospice Care: The Central Role of Autonomy for Informal Caregiver Resilience. J Family Nurs. 2019; 25(2):190218.

31. Lima AMN, Martins MMPS, Ferreira MSM, Coelho ARN, Schoeller SD, Parola VSO. Prática de enfermagem na promoção da autonomia dos idosos. Rev Esc Enferm USP. No prelo, 2021.

32. Valero IA. Autonomies in interaction: Dimensions of patient autonomy and nonadherence to treatment. Front Psychol. 2019; 10(AUG).

33. Reis MGM, Casa-Novas MV, Serra I, Magalhães MDDC, Sousa LMM. The importance of a training program on active aging from the perspective of elderly individuals. Rev Bras Enferm. 2021; 74:1-7.
34. Kirkscey
$\mathrm{R}$.
Bioethical communication: shared

decision-making and relational empathy. J Commun Health. 2018; 11(3):164-74.

35. Paton A. "Being Guided": What oncofertility Patients' decisions can teach us about the efficacy of autonomy, agency, and decision-making theory in the contemporary clinical encounter. Int J Fem Approaches to Bioeth. 2019; 12(2):18-35.

36. Roland T, Wimberger K, Amsuess S, Russold MF, Baumgartner W. An Insulated Flexible Sensor for Stable Electromyography Detection: Application to Prosthesis Control. Sensors. 2019; 19(4):961-1.

37. Sorinmade OA. Relevant information and the Mental Capacity Act. J Patient Saf. 2019; 24(2):71-5.

38. Delmar C. The interplay between autonomy and dignity: summarizing patients voices. Med Health Care Philos. 2013; 16(4):975-81. 
Financiamento: Os autores declaram que não houve financiamento.

Conflito de interesses: Os autores declaram não haver conflito de interesses.

Participação dos autores:

- Concepção: Lima AMN, Martins MMFS, Ferreira MSM, Fernandes CS, Schoeller SD, Rodrigues TMP, Parola VSO.

- Desenvolvimento: Lima AMN, Martins MMFS, Ferreira MSM, Fernandes CS, Schoeller SD, Rodrigues TMP, Parola VSO.

- Redação e revisão: Lima AMN, Martins MMFS, Ferreira MSM, Fernandes CS, Schoeller SD, Rodrigues TMP, Parola VSO.

Como citar este artigo: Lima AMN, Martins MMFS, Ferreira MSM, Fernandes CS, Schoeller SD, Rodrigues TMP, et al. Autoavaliação da promoção da autonomia dos idosos: um estudo Delphi. J Health NPEPS. 2021; 6(1):155-174.

Submissão: 23/12/2021

Aceito: $20 / 03 / 2021$

Publicado: 01/06/2021 